\title{
Ethical Considerations for Participatory Health through Social Media: Healthcare Workforce and Policy Maker Perspectives
}

\section{Contribution of the IMIA Participatory Health and Social Media Working Group}

\author{
Octavio Rivera-Romero ${ }^{1}$, Stathis Konstantinidis², Kerstin Denecke ${ }^{3}$, Elia Gabarrón ${ }^{4}$, \\ Carolyn Petersen ${ }^{5}$, Mowafa Househ ${ }^{6}$, Mark Merolli ${ }^{7}$, Miguel Ángel Mayer ${ }^{8}$ \\ 1 Computer Engineering School, Universidad de Sevilla, Seville, Spain \\ 2 University of Nottingham, Nottingham, UK \\ 3 Bern University of Applied Sciences, Bern, Switzerland \\ ${ }^{4}$ Norwegian Centre of E-Health Research, University Hospital North Norway, Norway \\ 5 Division of Biomedical Statistics and Informatics, Mayo Clinic, Rochester, MN, USA \\ ${ }^{6}$ College of Science and Engineering, Hamad Bin Khalifa University, Doha, Qatar \\ 7 Health and Biomedical Informatics Centre, Melbourne Medical School, University of Melbourne, \\ Melbourne, Australia \\ 8 Research Programme on Biomedical Informatics, Hospital del Mar Medical Research Institute, \\ Universitat Pompeu Fabra, Barcelona, Spain
}

\section{Summary}

Objectives: To identify the different ethical issues that should be considered in participatory health through social media from different stakeholder perspectives (i.e., patients/service users, health professionals, health information technology (IT) professionals, and policy makers) in any healthcare context. Methods: We implemented a two-round survey composed of open ended questions in the first round, aggregated into a list of ethical issues rated for importance by participants in the second round, to generate a ranked list of possible ethical issues in participatory health based on healthcare professionals' and policy makers' opinions on both their own point of view and their beliefs for other stakeholders' perspectives.
Results: Twenty-six individuals responded in the first round of the survey. Multiple ethical issues were identified for each perspective. Data privacy, data security, and digital literacy were common themes in all perspectives. Thirty-three individuals completed the second round of the survey. Data privacy and data security were ranked among the three most important ethical issues in all perspectives. Quality assurance was the most important issue from the healthcare professionals' perspective and the second most important issue from the patients' perspective. Data privacy was the most important consideration for patients/service users. Digital literacy was ranked as the fourth most important issue, except for policy makers' perspective.
Conclusions: Different stakeholders' opinions fairly agreed that there are common ethical issues that should be considered across the four groups (patients, healthcare professionals, health IT professionals, policy makers) such as data privacy, security, and quality assurance.

\section{Keywords}

Social media; ethical issues; clinical ethics; patient-centered care; patient participation

Yearb Med Inform 2020:71-6

http://dx.doi.org/10.1055/s-0040-1701981

\section{Introduction}

Nowadays, individuals have more autonomy, access to information, and human capital to support their health decisions than previously fathomable [1,2]. These informed, connected, and socially supported health consumers (or patients) are leading a shift in the way healthcare is approached, delivered, and governed. This very notion lies at the heart of participatory health, which centers on collaboration and shared-decision making $[2,3]$.
Social media and other collaborative technologies found their role in healthcare by providing tools to deliver participatory health services. Social media are defined as platforms that foster information exchange and the creation of user-generated content [4, 5]. They are arguably the best-known example of the way in which digital healthcare has shaped the participatory health paradigm. They are being used to support healthcare delivery [6], health information search and delivery [6, 7], connection with like-minded individuals, and sharing and crowdsourcing experiences [5-6, 8-9], or promotion of healthy behaviors $[10,11]$.

Despite increasing prevalence and potential to support care, the pace at which these platforms are evolving is rapid but guidance/ policies surrounding their best practice use are slow to keep up [6]. So too is our understanding of adoption-related factors and intentions for use [6]. While it is rela- 
tively straightforward to conceptualize social media use for the purposes of information sharing, connection, and communication, these platforms have quickly evolved beyond their original intended purposes. They have become platforms for research conduct (e.g., participant recruitment), mass data collection and exchange, and surveillance of disease or adverse events [12-15]. This situation becomes even more complex when advances in artificial intelligence are taken into consideration [16]. Social networks have become a common platform for researchers to monitor, mine, and analyze observational data about health $[17,18]$.

Ethics in digitalized healthcare is becoming increasingly a hot topic. Currently, there are attempts to define guiding principles for the creation and use of health information and applications on the Internet [19] and governments' regulations such as the recent EU General Data Protection Regulation (GDPR) [20]. According to systematic evidence, there is a need for guidelines for professional accountability in e-health service delivery [21] and ethical frameworks that stimulate passive data-driven innovation while protecting patient integrity [22]. Ethical concerns still need to be addressed.

Due to social media not being first conceived as platforms for research (e.g., health data collection and data exchange), policies and legislation have been slow to keep up $[9,14,23]$. As informaticians, clinicians, IT professionals, researchers, and policy makers with expertise and interest in health social media extend and expand their use of social media, the ethical debate has never been more prudent. Unethical practices by major social media platforms, such as misuse and manipulation of data, have been a major area of attention in recent times, have been at the forefront of the media, and have dominated major policy debates [24,25]. It is not unreasonable to connect these issues directly to the healthcare domain; as health data are arguably among the most sensitive data

Unpacking and applying several ethical principles to health social media research remains a challenge for participatory health informatics researchers, but equally for health and IT professionals, and for policy makers [14, 26, 27]. We must all understand and pay special attention to ethical and trust-related considerations, such as privacy, confidentiality, consent, and cybersecurity $[18,28]$. Terrasse et al., discussed the impact of social networking sites on the doctor-patient relationship, the development of e-health platforms to deliver care, the use of online data and algorithms to inform health research, and the broader public health consequences of widespread social media use resulting in a list of recommendations [29]. It would seem that on the whole, concerns about social media use center mostly on "uncertainty" about the legal implications of use [23]. Both Azer and Terrasse et al., $[23,30]$ raised the need to better determine the ethical process in studies that include the use or the creation of social media technical implementations.

Despite increased interest, prevalence, and uptake, there is still limited evidence and commentary surrounding these ethical considerations for engaging with social media from the perspectives of various stakeholders. Members of the current authorship team (who are members of the International Medical Informatics Association (IMIA) Participatory Health and Social Media Working Group), have previously published research on ethical issues related to social media in healthcare to start exploring the above questions $[28,31]$. In this previous work, they provided a preliminary look into various ethical issues/scenarios that should be considered when engaging in social media use for participatory health in particular contexts (e.g., special populations, patient-practitioners communication, crowdsourcing, research conduct, among others). However, that work did not contextualize and quantify ethical issues in social media use from the specific perspectives of different stakeholder groups (e.g., service users, clinicians, IT professionals, and policy makers). It is these perspectives that we explore here to build upon the earlier work.

Hence, the aim of this study is to identify the different ethical issues that should be considered in participatory health through social media for patients/service users, health professionals, health IT professionals, and policy makers from different stakeholders' perspectives, including health professionals, health IT professionals, and policy makers, in any healthcare context.

\section{Methods}

In order to identify the different ethical issues, we used a two-round survey. In the first round, a set of four open-ended questions allowed the participants to provide qualitative data regarding the ethical issues that should be considered in participatory health through social media, according to their own point of view, and also considering the perspectives from the following four different stakeholders' perspectives: patient/service user/general public, healthcare professional (including clinical and allied health professions), health IT professionals (health informaticians, etc.), and policy makers. The four questions were created and reviewed by all the authors of this study. Authors aggregated the results of the first round into a survey for each of the above stakeholders' category that formed the round two surveys. The round two survey started from the identified and aggregated ethical issues for each of the above categories. Then participants were asked to rate each identified ethical issue for each of the four categories using a five-point Likert scale with options label from not important (1) to very important (5). Review and approval for the study was obtained from the Andalusian Ethics Committee of the Biomedical Research (n. 1504-N-19).

A purposive sampling method was followed. Eligible participants for both surveys were stakeholders of participatory health excluding patients or service users, including but not limited to clinicians, nurses, allied health professionals, and health IT professionals. Furthermore, participants included researchers, developers, policy makers, and members of professionals' associations. Unless there was a contact with researchers, the pool of participants was the same in both rounds.

Participant recruitment was conducted through email to foster anonymity. Initially an email message was disseminated by the chief investigator of the study, and all authors forwarded it to their peer networks. In the first round, participants were given two weeks to respond, with a reminder email sent weekly, while in the second round participants were given one week to respond.

For each of the four stakeholder groups of the first round, two different authors thematically analyzed the results, while three 
of the authors reviewed and agreed upon the selected results. In the second round, the final prioritization was conducted following recommendations enabling the strength of "importance" [32]. Each scored survey ethical issue's rating value was multiplied by the frequency of each rating of this issue. The final ethical issues per stakeholders' category were assigned an "importance" rank.

\section{Results}

\subsection{First Round Survey Results}

The first round of the survey was answered by 26 respondents with an average of 16.9 years of professional experience (minimum $=0.5$, maximum $=40, \mathrm{SD}=10.5$ ). Demographics are shown in Table 1 included in Appendix II. Participants in the first round were from the United States (34.6\%), Europe (61.5\%), and Qatar (3.9\%), with 34.6\% of health professionals, $50 \%$ of health IT professionals, $19.2 \%$ of policy makers, and $50 \%$ of other professionals (academics, researchers, and one attorney). Participants' responses were coded and aggregated by the authors to a list of ethical issues for each category of stakeholders (see Appendix II) to be used in the second round of the survey.

According to participant professionals' opinion, there are multiple ethical issues that should be considered from the patients' or general public's perspective. A theme was around digital literacy of patients or general public that results in a gap for understanding what is happening with the data shared. Risks for patients were central to participants' views considering the potentiality of re-identification of sensitive data, reliability of provided information, and commercialization of data, which was noted in multiple responses.

Regarding the healthcare professionals' perspective, ethical issues around their responsibility against patients or the general public were raised, including awareness of risks, lack of clarity to integrate information from these channels into patient care, and potential for blurring professional boundaries. Digital literacy of healthcare professionals that might result in risks to patients was raised by participants. Data use and reuse, including data confidentiality, data ownership, and commercialization of sensitive data, were also identified as a potential ethical issue by respondents.

From the health IT professionals' perspective, core to the participants' perspective was the ethical issues around data collection and use, and the need for transparency. Participants noted that ethical issues might arise due to lack of consideration of users' cultures (of participatory health technologies) since they are reflected both in user interfaces and in the algorithms used.

There are many ethical issues from the perspective of policy makers that need to be considered when trying to plan, implement, and evaluate participatory health-related technologies. The responses highlighted the need for policy makers to be cognizant of the impacts of participatory health technologies on vulnerable populations and to understand the ethical issues relevant to patients' risks and benefits, as well as the digital divide between patients. Furthermore, ethical issues around data use might lead to the misuse through commercialization of the data or the ability to re-identify patients. Lack of digital literacy of policy makers might lead to ethical considerations by putting patients or the general public at risk and/or by not applying responsible innovation to participatory health platforms, as one respondent noted.

The above analysis was used in the development of the questions for the second round of the survey. As an example, the thematic analysis for the policy makers' perspective can be found in the appendix III.

\subsection{Second Round Survey Results}

The second round of the survey was answered by 33 individuals, with an average of 15.7 years of professional experience ( minimum $=3$, maximum $=40, \mathrm{SD}=10.2$ ). Demographics are shown in Table 4 included in Appendix IV. Participants were from the United States (24.3\%), Europe (45.6\%), Qatar (12.1\%), Australia (3\%), and Israel (3\%), with $57.6 \%$ of health professionals, $48.5 \%$ of health IT professionals, $9.1 \%$ of policy makers, and $39.4 \%$ of other professionals (academics and researchers). Fifteen of the respondents of the second round had participated to the first round of the survey (45.5\%). Appendix IV includes tables summarizing detailed information regarding those results (Figure 1).

When it comes to the ethical issues for patients or the general public, more than three-quarters of the respondents consider that quality assurance, data privacy, data security, ownership of the data, and education for patients are absolutely essential or very important. Ethical issues that were found to be of minor importance for patients or the general public were: (1) Data ownership (noted by seven respondents: two nurses; two researchers, one professor, one IT professional, and one managing director/ CEO); (2) Patient education (noted by five respondents: two nurses, two researchers, and one managing director/CEO); and (3) Data security (noted by four respondents: one physiotherapist, and three researchers). Both quality assurance and data privacy are reported as moderately important by one respondent (an IT professional in both cases).

Regarding the ethical issues for healthcare professionals, quality assurance, data privacy, data security, and education of healthcare professionals are highlighted by more than $80 \%$ of respondents as absolutely or very important aspects. On the other hand, two issues are considered of less importance by a higher proportion of respondents: (1) Data ownership, ranked as moderately/slightly important by eight respondents (two researchers, two managing director/CEO, one nurse, one medical doctor, one professor, and one IT professional) and as not important at all by one researcher, followed by (2) Work overload for healthcare professionals, considered of moderate or slightly importance by eight respondents (three professors, two researchers, one nurse, one physiotherapist, and one product manager). Responsible use is found to be of moderate importance for healthcare professionals by four respondents (two professors, one researcher, and one nurse).

With regard to the ethical aspects of importance for IT professionals, more than three quarters of respondents note that data privacy, data security, and responsible use are absolutely or very important. Ethical issues that are considered of lowest importance by a higher number of respondents are: (1) Education of IT professionals, ranked as of moderate importance by seven individuals 


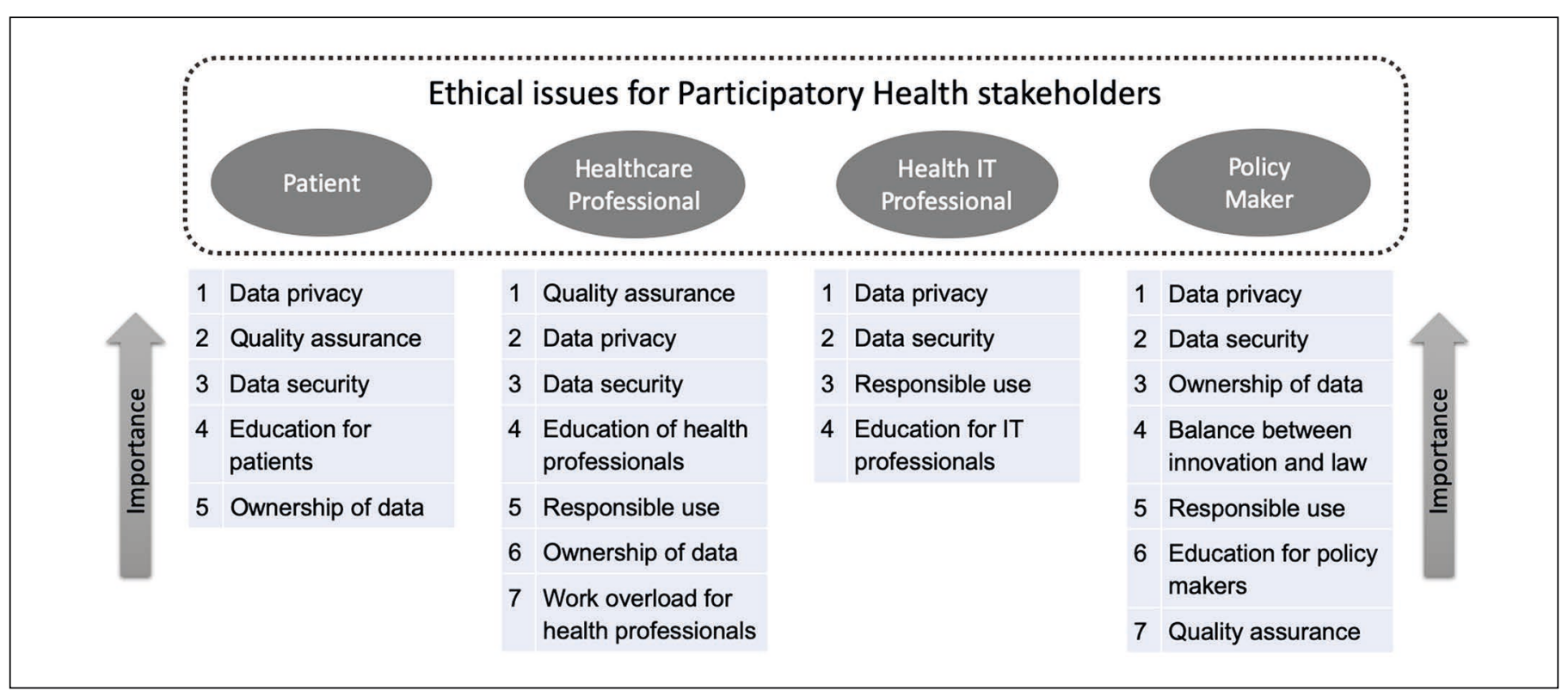

Fig. 1 Overview of Ethical Issues for Participatory Health Stakeholders

(three researchers, two medical doctors, one professor, and one managing director/ CEO), followed by (2) Responsible use, also considered of moderate importance by seven respondents (two researchers, two medical doctors, one professor, and one managing director/CEO).

All the ethical issues linked to the use of social media by policy makers that were raised in the first round of the survey are considered absolutely essential or very important by more than three-quarters of the respondents in the second round.

\section{Discussion}

\subsection{Data Privacy, Security, and Quality Assurance are the Most Relevant Ethical Issues}

Data privacy, security, and quality assurance are regarded as the most relevant from both patient/service users' and healthcare professionals' perspectives. From the healthcare professionals' perspective, quality might have two facets: ensuring the quality of data provided by healthcare professionals (e.g., in forums, blogs, or other social media), and the quality of data about a patient considered in the patient's treatment. Our study does not provide hints on which facet is meant by the survey participants. From the patients' perspective, the quality of the consumed social media content is relevant because it might have direct effects on patients' decision-making or perhaps psychological implications (e.g., negative implications such as becoming reluctant to undergo a therapy because of fear, or positive implications such as encouragement for behavior change). Furthermore, it might lead to health misinformation, including spread of inaccurate or false information (e.g., antivaccination [32], anorexia promotion [33], faulty claims about dietary product and drugs [34]). However, the facet "quality of the provided content" might also be relevant from the patients' perspective in that error-prone data used by healthcare professionals in planning treatment might create patient harm. A third perspective of quality assurance is ensuring quality of healthcare services using social media. The findings presented in this manuscript have practical clinical implications for the therapeutic relationship, whereby health professionals are comfortable in recommending social media use for their patients [35]. Secondly, if patients' (and physicians') statements and comments about the quality of healthcare services can be analyzed, these can be transformed into quality indicators to measure healthcare performance and make improvements [36].

The awareness of data privacy and security issues related to participatory health is obvious from our study results, and it can be inferred that this awareness increased in recent years [37]. This might be due to recent data scandals such as the Facebook-Cambridge Analytica scandal and the efforts to establish laws trying to prevent such scandals. In Europe, the General Data Protection Regulation (GDPR) came into force in May 2018 and improved legislation of how persons should be protected with regards to the processing of personal data about them. The GDPR applies to the data collection performed by private marketing companies as well as to research by private companies or public universities in all EU member states [38]. One goal of the GDPR is to "protect the fundamental rights and freedoms of the data subjects by creating a protective regiment with regards to the processing of personal data" [38]. This regulation clearly affects social media research and participatory health. From the social media researchers' perspective, methods must be established permitting social media users to 
request the removal of their data, which could affect the results. Practically speaking, it is nearly impossible to guarantee anonymity, in that when more data about an individual becomes available it might reveal patterns of behavior or characteristics that could reveal the individual's identity. Research has shown that the willingness to participate in biomedical research advertised on social media platforms and search engines, as well as the willingness to share digital data with researchers, have been affected by recent news on the misuse of such data [39]. However, research about willingness to share data in health social media is still in its infancy and hence, further research in this space within a participatory health paradigm is warranted [40].

Because the sharing of personal health data requires patients' consent, which can be complicated in participatory health through social media [41], initiatives to educate individuals about the benefits of sharing health data have been used in some instances. For example, the initiative "Understanding Patient Data" (https:// understandingpatientdata.org.uk) promotes improvements in informing patients on the use of their health information. The work aims to explain to patients how and why data can be used for care and research to inform about duties and rights, and how personal information is kept safe.

\subsection{Both Patients' and Healthcare Professionals' Education Are Relevant Issues too}

An interesting result in our study is that education of health professionals and patients is considered relevant in the context of social media and participatory health. This has clinical implications because education of patients might include teaching them applicable legal rights with respect to their data privacy. In particular, rights conferred by the GDPR could be of interest. Providing consent requires an understanding of possible consequences of data usage and sharing, which in turn requires transparency about potential data use and transmission by social media researchers, healthcare professionals using such data, and social media providers.
In contrast to other works on the limitations and benefits of social media for participatory health, our study participants did not consider negative impacts of social media on the health and mental well being of patients. Such harmful effects include increased exposure to social isolation, depression and cyber-bullying, and triggering of addictive behaviors with losses in self-efficacy and confidence $[42,43]$.

\subsection{Strengths and Limitations}

We explicitly included in this research only health professionals, IT professionals, and policy makers (i.e., persons who are either developing technologies for participatory health and social media, those who contribute or analyze the data in a professional manner for healthcare purposes, or those involved in legislation and regulation). Including patients/service users' perspectives might result in a better representative list of ethical issues for this group. We recruited participants from all over the world. Even though the study ran only for a short period of time, responses were received from individuals in multiple European countries, the United States, and Australia. This allowed us to get input from different perspectives, which nonetheless turned out to be quite similar. However, it is possible that these perspectives are less similar than reflected in the results because respondents in different countries may identify the names of their roles differently.

The questionnaire was distributed through the networks of the authors of this paper. This method may have introduced a bias because the authors might inadvertently have selected the persons with whom the questionnaire was shared. Furthermore, there was no participant from developing countries, which views could have differently shaped the findings of this study. As a result, it is unclear whether a representative sample was achieved. All authors of this paper are linked with researchers, policy makers, IT and healthcare professionals all over the world. Therefore, we believe that the respondents' answers reflect a broad view of the topic.

\section{Conclusion}

In this paper we describe a two-round survey conducted to identify ethical issues that should be considered by different stakeholders (patients, healthcare professionals, health IT professionals, policy makers) from the perspective of the wider healthcare field (health professionals, policy makers, health IT professionals) without excluding researchers in the field, CEOs from relevant companies, and anyone considering she/he is an expert on the topic.

Different stakeholders' opinions fairly agreed that there are common ethical issues that should be considered across the four groups (patients, healthcare professionals, health IT professionals, policy makers) including data privacy, security, and quality assurance. All of these are applicable across clinical practices and researchers. However, according to stakeholders' views each individual group should consider additional ethical issues and with different importance (Figure 1). Future research from the direct perspective of the health consumers' voice is warranted.

This study neither focused on ethical issues arising from specific participatory technologies nor on specific contexts, but aimed to give a wider perspective on ethical issues as seen by health professionals. Despite the limitations of the current study, the authors believe that it raises awareness of a number of ethical issues that each health-related profession should pay attention to, and it provides a prioritized list of ethical issues for consideration in the practice setting and when conducting research in health social media. As alluded to, the implications of this list are many, and can act as the basis for future in-depth research on each ethical issue as well as become the basis for curricula or training programs for patients, health professionals, health IT professionals, and policy makers.

\section{References}

1. deBronkart D. From patient centred to people powered: autonomy on the rise. BMJ [Internet] 2015 Feb 10 [cited 2019 Nov 28];350:h148. Available from: http://www.ncbi.nlm.nih.gov/ pubmed $/ 25670184$

2. Finset A. Patient Participation, Engagement and Activation: Increased emphasis on the role of 
patients in healthcare. Patient Educ Couns 2017 Jul 1;100(7):1245-6.

3. Millenson ML. When "patient centred" is no longer enough: The challenge of collaborative health: An essay by Michael L Millenson. BMJ 2017;358.

4. Eysenbach G. Medicine 2.0: Social networking, collaboration, participation, apomediation, and openness. J Med Internet Res 2008;10(3):e22.

5. Grajales FJ, Sheps S, Ho K, Novak-Lauscher H, Eysenbach G. Social media: A review and tutorial of applications in medicine and health care. J Med Internet Res 2014;16(2):e13.

6. Hazzam J, Lahrech A. Health care professionals' social media behavior and the underlying factors of social media adoption and use: Quantitative study. J Med Internet Res 2018 Nov 1;20(11).

7. Merolli M, Gray K, Martin-Sanchez F, Mantopoulos S, Hogg M. Using Social Media While Waiting in Pain: A Clinical 12-Week Longitudinal Pilot Study. JMIR Res Protoc 2015 Aug 7;4(3):e101.

8. Moorhead SA, Hazlett DE, Harrison L, Carroll $\mathrm{JK}$, Irwin A, Hoving C. A new dimension of health care: Systematic review of the uses, benefits, and limitations of social media for health communication. J Med Internet Res 2013;15(4):e85.

9. Carlquist E, Lee NE, Shalin SC, Goodman M, Gardner JM. Dermatopathology and social media a survey of 131 medical professionals from 29 countries. Arch Pathol Lab Med 2018 Feb 1;142(2):184-90.

10. Gabarron E, Wynn R. Use of social media for sexual health promotion: A scoping review. Glob Health Action 2016;9(1)

11. Gabarron E, Arsand E, Wynn R. Social media use in interventions for diabetes: Rapid evidence-based review. J Med Internet Res 2018;(8): e10303.

12. Collier N, Son NT, Nguyen NM. OMG U got flu? Analysis of shared health messages for bio-surveillance. J Biomed Semantics 2011 Oct 6;2(5).

13. Swan M. Crowdsourced health research studies: An important emerging complement to clinical trials in the public health research ecosystem. J Med Internet Res 2012;14(2):186-98.

14. Nikolic A, Wickramasinghe N, Claydon-Platt D, Balakrishnan V, Smart P. The use of communication apps by medical staff in the australian health care system: Survey study on prevalence and use. J Med Internet Res 2018; 6(1):e9.

15. Bender JL, Cyr AB, Arbuckle L, Ferris LE. Ethics and privacy implications of using the internet and social media to recruit participants for health research: A privacy-by-design framework for online recruitment. J Med Internet Res 2017 Apr 1;19(4).

16. Denecke K, Gabarron E, Grainger R, Konstantinidis ST, Lau A, Rivera-Romero O, et al. Artificial Intelligence for Participatory Health: Applications, Impact, and Future Implications. Yearb Med Inform 2019:165-73.

17. Rafla M, Carson NJ, DeJong SM. Adolescents and the Internet: What Mental Health Clinicians Need to Know. Curr Psychiatry Rep 2014;16(9):472.

18. Golder S, Scantlebury A, Christmas H. Understanding Public Attitudes Toward Researchers Using Social Media for Detecting and Monitoring
Adverse Events Data: Multi Methods Study. J Med Internet Res 2019 Aug 29;21(8):e7081.

19. Rippen H, Risk A; e-Health Ethics Initiative. e-Health Code of Ethics (May 24). J Med Internet Res 2000;2(2):e9.

20. European Union (Communities OJE). Regulation 2016/679 of the European parliament and the Council of the European Union; 2016.

21. Marziali E, Serafini JMD, McCleary L. A Systematic Review of Practice Standards and Research Ethics in Technology-Based Home Health Care Intervention Programs for Older Adults. J Aging Health [Internet] 2005 Dec 30 [cited 2019 Nov 28];17(6):679-96. Available from: http://journals. sagepub.com/doi/10.1177/0898264305281100

22. Maher NA, Senders JT, Hulsbergen AFC, Lamba N, Parker M, Onnela JP, et al. Passive data collection and use in healthcare: A systematic review of ethical issues. Int J Med Inform 2019;129:242-7.

23. Brown J, Ryan C, Harris A. How doctors view and use social media: A national survey. J Med Internet Res 2014 Dec 1;16(12).

24. Kleinsman J, Buckley S. Facebook Study: A Little Bit Unethical But Worth It? J Bioeth Inq 2015 Jun 1;12(2):179-82.

25. Esteve A. The business of personal data: Google, Facebook, and privacy issues in the EU and the USA. Int Data Priv Law 2017;7(1):36-47.

26. Chretien KC, Kind T. Social media and clinical care: Ethical, professional, and social implications. Circulation 2013 Apr 2;127(13):1413-21.

27. Horvitz E, Mulligan D. Data, privacy, and the greater good. Science 2015 Jul 17;349(6245):253-5.

28. Denecke K, Bamidis P, Bond C, Gabarron E, Househ M, Lau AYS, et al. Ethical Issues of Social Media Usage in Healthcare. Yearb Med Inform 2015:137-47.

29. Terrasse M, Gorin M, Sisti D. Social Media, E-Health, and Medical Ethics. Hastings Cent Rep [Internet] 2019 Jan [cited 2019 Nov 28];49(1):24 33. Available from: http://doi.wiley.com/10.1002/ hast. 975

30. Azer SA. Social media channels in health care research and rising ethical issues. AMA J Ethics 2017;19(11):1061-9.

31. Denecke K. Ethical aspects of using medical social media in healthcare applications. Stud Health Technol Inform 2014;198:55-62.

32. Broniatowski DA, Jamison AM, Qi S, AlKulaib L, Chen T, Benton A, et al. Weaponized Health Communication: Twitter Bots and Russian Trolls Amplify the Vaccine Debate. Am J Public Health [Internet] 2018 Oct 1 [cited $2020 \mathrm{Feb}$ 27];108(10):1378-84. Available from: http://www. ncbi.nlm.nih.gov/pubmed/30138075

33. Syed-Abdul S, Fernandez-Luque L, Jian WS, Li YC, Crain S, Hsu MH, et al. Misleading health-related information promoted through video-based social media: Anorexia on youtube. J Med Internet Res 2013 Feb;15(2).

34. Al Khaja KAJ, AlKhaja AK, Sequeira RP. Drug information, misinformation, and disinformation on social media: a content analysis study. J Public Health Policy [Internet] 2018 Aug 1 [cited 2020 Feb 27];39(3):343-57. Available from: http://www. ncbi.nlm.nih.gov/pubmed/29795521

35. De Angelis G, Wells GA, Davies B, King J, Shallwani SM, McEwan J, et al. The use of social media among health professionals to facilitate chronic disease self-management with their patients: A systematic review. Digit Heal [Internet] 2018 Jan 3 [cited 2020 Feb 27];4:205520761877141. Available from: http://journals.sagepub.com/ doi/10.1177/2055207618771416

36. Greaves F, Ramirez-Cano D, Millett C, Darzi A, Donaldson L. Harnessing the cloud of patient experience: Using social media to detect poor quality healthcare. BMJ Qual Saf 2013;22(3):251-5.

37. Househ M, Borycki E, Kushniruk A. Empowering patients through social media: the benefits and challenges. Health Informatics J [Internet] 2014 Mar 18 [cited 2020 Feb 27];20(1):50-8. Available from: http://www.ncbi.nlm.nih.gov/ pubmed $/ 24550564$

38. Kotsios A, Magnani M, Rossi L, Shklovski I, Vega D. An Analysis of the Consequences of the General Data Protection Regulation (GDPR) on Social Network Research. 2019 Mar 7 [cited 2019 Nov 28]; Available from: http://arxiv.org/abs/1903.03196

39. Pratap A, Allred R, Duffy J, Rivera D, Lee HS, Renn BN, et al. Contemporary Views of Research Participant Willingness to Participate and Share Digital Data in Biomedical Research. JAMA Netw open 2019 Nov 1;2(11):e1915717.

40. Staccini P, Lau AYS. Findings from 2017 on Consumer Health Informatics and Education: Health Data Access and Sharing [Internet] Yearb Med Inform 2018 [cited 2020 Feb 27]: 163-9. Available from: http://www.thieme-connect.de/ DOI/DOI?10.1055/s-0038-1641218

41. Courbier S, Dimond R, Bros-Facer V. Share and protect our health data: An evidence based approach to rare disease patients' perspectives on data sharing and data protection - Quantitative survey and recommendations [Internet]. Vol. 14, Orphanet J Rare Dis 2019 [cited 2020 Feb 27];14(1): 175. Available from: https://ojrd.biomedcentral.com/ articles/10.1186/s13023-019-1123-4

42. Giustini DM, Ali SM, Fraser M, Boulos MNK. Effective uses of social media in public health and medicine: a systematic review of systematic reviews. Online J Public Health Inform 2018 Sep $21 ; 10(2)$.

43. Song H, Zmyslinski-Seelig A, Kim J, Drent A, Victor A, Omori K, et al. Does Facebook make you lonely?: A meta analysis. Comput Human Behav [Internet] $2014 \mathrm{Jul}$ [cited 2019 Nov 28];36:446-52. Available from: https://inkinghub.elsevier.com/ retrieve/pii/S0747563214002209

\section{Correspondence to:}

Octavio Rivera Romero

Avda Reina Mercedes s/n

ETSI Informática, G1.43 Seville (Spain)

Tel: + 34954552789

Fax: +34 954552764

E-mail: orivera@us.es 